\title{
PERMUTATION GROUPS WITH PROJECTIVE UNITARY SUBCONSTITUENTS
}

\author{
RICHARD WEISS
}

\begin{abstract}
Let $\Gamma$ be a finite directed graph with vertex set $V(\Gamma)$ and edge set $E(\Gamma)$ and let $G$ be a subgroup of aut $(\Gamma)$ which we assume to act transitively on both $V(\Gamma)$ and $E(T)$. Suppose that for some prime power $q$, the stabilizer $G(x)$ of a vertex $x$ induces on both $\{y \mid(x, y) \in E(\Gamma)\}$ and $\{w \mid(w, x) \in E(\Gamma)\}$ a group lying between $\operatorname{PSU}\left(3, q^{2}\right)$ and $P \Gamma U\left(3, q^{2}\right)$. It is shown that if $G$ acts primitively on $V(\Gamma)$, then for each edge $(x, y)$, the subgroup of $G(x)$ fixing every vertex in $\{w \mid(x, w)$ or $(y, w) \in$ $E(\Gamma)\}$ is trivial.
\end{abstract}

Let $\Gamma$ be a directed graph with vertex set $V(\Gamma)$ and edge set $E(\Gamma)$ and let $G$ be a subgroup of aut $(\Gamma)$ which we assume to act transitively on both $V(\Gamma)$ and $E(\Gamma)$. Let $x \in V(\Gamma)$ be arbitrary. We denote by $\Gamma(x)$ the set of $y \in V(\Gamma)$ such that $(x, y) \in$ $E(\Gamma)$ and by $\Gamma^{\prime}(x)$ the set of $w \in V(\Gamma)$ such that $(w, x) \in E(\Gamma)$. Since $G$ acts transitively on $E(\Gamma)$, the stabilizer $G(x)$ of $x$ in $G$ acts transitively on both $\Gamma(x)$ and $\Gamma^{\prime}(x)$ and either $\Gamma(x) \cap \Gamma^{\prime}(x)=\varnothing$ or $\Gamma(x)=\Gamma^{\prime}(x)$. In the latter case, we may identify $\Gamma$ with the undirected graph with vertex set $V(\Gamma)$ and edge set $\{\{x, y\} \mid(x$, $y) \in E(\Gamma)\}$ and will, in fact, simply say that $\Gamma$ itself is undirected. Let $G_{1}(x)=\{a$ $\in G(x) \mid a \in G(y)$ for all $y \in \Gamma(x)\}$ and $G_{1}^{\prime}(x)=\{a \in G(x) \mid a \in G(w)$ for all $w \in$ $\left.\Gamma^{\prime}(x)\right\}$. For $u \in \Gamma(x)$ or $\Gamma^{\prime}(x)$, we set $G(x, u)=G(x) \cap G(u)$ and $G_{1}(x, u)=G_{1}(x)$ $\cap G_{1}(u) . \Gamma$ is called connected if for every two vertices $u$ and $v$, there is a sequence $\left(x_{0}, x_{1}, \ldots, x_{s}\right)$ of vertices such that $x_{0}=u, x_{s}=v$ and $x_{i-1} \in \Gamma\left(x_{i}\right) \cup \Gamma^{\prime}\left(x_{i}\right)$ for $1<i<s$. The following observation is easily verified.

LEMMA 1. If $\Gamma$ is connected and undirected and $\{x, y\} \in E(\Gamma)$, then $\langle G(x), G(y)\rangle$ acts transitively on $E(\Gamma)$.

Note that this statement does not hold if we do not assume $\Gamma$ to be undirected. Consider, for instance, the graph $\Gamma$ with $V(\Gamma)=\mathbf{Z}_{k} \times M, M$ an arbitrary nonempty set and $k \geqslant 3$, and $E(\Gamma)=\{((i, x),(j, y)) \mid i-j \equiv 1(\bmod k)\}$ and $G=$ $\operatorname{aut}(\Gamma)$.

If $G$ is an arbitrary transitive permutation group on a set $\Omega$ and $\Delta$ an orbit of $G$ on $\Omega \times \Omega$ (called an orbital of $G$ ), then $G$ can be considered as a vertex- and edge-transitive subgroup of $\operatorname{aut}\left(\Gamma_{\Delta}\right)$ where $\Gamma_{\Delta}$ is the graph with vertex set $\Omega$ and edge set $\Delta$. According to [9, (4.4)], $G$ is primitive on $\Omega$ if and only if, for each nondiagonal orbital $\Delta, \Gamma_{\Delta}$ is connected.

Received by the editors April 21, 1978 and, in revised form, September 21, 1978.

AMS (MOS) subject classifications (1970). Primary 20B15, 20B25; Secondary 05C25.

Key words and phrases. Primitive permutation group, subconstituent, projective unitary group, symmetric graph. 
In [2] and [3], Dempwolff proved the following result.

TheOREM 2. Let $q$ be a prime power, $q>2$. Let $\Gamma$ be a finite graph and $(x, y) \in E(\Gamma)$ arbitrary. Suppose $\operatorname{aut}(\Gamma)$ contains a subgroup $G$ acting transitively on both $V(\Gamma)$ and $E(\Gamma)$ such that both $G(x)^{\Gamma(x)}$ (i.e., the permutation group induced by $G(x)$ on $\Gamma(x))$ and $G(x)^{\Gamma^{\prime}(x)}$ are isomorphic to $\operatorname{PSU}\left(3, q^{2}\right)$ in its usual 2-transitive representation of degree $q^{3}+1$. Suppose further that $G$ is primitive on $V(\Gamma)$ or that $\Gamma$ is undirected and connected. Then $G_{1}(x, y)=1$.

Actually, Dempwolff simply required $G$ to be primitive on $V(\Gamma)$. This hypothesis is used, however, only in the following way.

Suppose $H$ is a subgroup of $G(x, y)$ normalized by both $G(x)$ and $G(y)$. Since $G$ is primitive and not regular on $V(\Gamma), G=\langle G(x), G(y)\rangle$. Hence $H \triangleleft G$. Since $H$ fixes the edge $(x, y), H$ fixes every edge. Therefore $H=1$.

In light of Lemma 1 , this same conclusion (i.e., $H=1$ ) holds if, instead of assuming $G^{V(\Gamma)}$ to be primitive, we assume $\Gamma$ to be undirected and connected.

In this paper, we prove the following improved version of Theorem 2.

THEOREM 3. Let $q$ be an arbitrary prime power. Let $\Gamma$ be a finite graph and $(x, y) \in E(\Gamma)$ arbitrary. Suppose aut $(\Gamma)$ contains a subgroup $G$ acting transitively on both $V(\Gamma)$ and $E(\Gamma)$ such that $\operatorname{PSU}\left(3, q^{2}\right) \leqslant G(x)^{\Gamma(x)} \leqslant P \Gamma U\left(3, q^{2}\right)$ and $P S U\left(3, q^{2}\right) \approx G(x)^{\Gamma^{\prime}(x)} \leqslant P \Gamma U\left(3, q^{2}\right)$. Suppose that $G$ is primitive on $V(\Gamma)$ or that $\Gamma$ is undirected and connected. Then $G_{1}(x, y)=1$.

Our proof of Theorem 3, although similar to the proof of Theorem 2 given in [2] and [3], is more elementary in that we avoid having to prove [2, (2.3)] and [3, (2.5)]. Moreover, our proof remains valid with only the most minor changes when we replace $P S U\left(3, q^{2}\right)$ by $S z(q)$ or ${ }^{2} G_{2}(q)$ (and $P \Gamma U\left(3, q^{2}\right)$ by the corresponding automorphism group) in the statement of Theorem 3 once it is shown that $S z(q)$ and ${ }^{2} G_{2}(q)$ have a certain elementary property. For $S z(q)$, this property is easily checked; for ${ }^{2} G_{2}(q)$, verification is more difficult. See the remarks at the end of our proof of Theorem 3. Of course, these versions of Theorem 3 were already proved in $[1,(2.6)$ and (3.5)], but only by using the very deep results [4, Corollary 10] in the case $S z(q)$ and $[5,(1.4)]$ in the case ${ }^{2} G_{2}(q)$.

We begin the proof of Theorem 3 by gathering the properties of $\operatorname{PSU}\left(3, q^{2}\right)$ which will be needed. The reader unacquainted with these groups is referred to [7] (in particular, pp. 242-244). Let $\Pi$ be the desarguesian plane $P G\left(2, q^{2}\right)$ over the field $G F\left(q^{2}\right), \delta$ the unitary polarity of $\Pi$ corresponding to a nondegenerate hermitian form on the underlying vector space and $X$ the set of absolute points (i.e., those $x$ in $\Pi$ incident with $\left.x^{\delta}\right) . \operatorname{PSU}\left(3, q^{2}\right)$ is the subgroup of $\operatorname{PSL}\left(3, q^{2}\right)$ consisting of those elements which commute with $\delta$. Let $H=P S U\left(3, q^{2}\right) . H$ acts 2-transitively on $X$ and $|X|=q^{3}+1$. Let $x \in X$ be arbitrary. Let $q=p^{n}, p$ prime. Then $O_{p} H(x)$ (i.e., the largest normal $p$-subgroup of the stabilizer $H(x)$ ) acts regularly on $X-\{x\}$ and its center $Z O_{p} H(x)$, which is of order $q$, consists of precisely those elements of $H(x)$ fixing all the $q^{2}$ nonabsolute lines (i.e., those lines $L$ of $\Pi$ not incident with $L^{\delta}$ ) passing through $x$. It is straightforward to check by calculating with the elements denoted by $Q(a, b)$ and $T$ in [7, pp. 243-244] that if 
$x_{1}, x_{2}$ and $x_{3} \in X$ are noncollinear, then $\left\langle Z O_{p} H\left(x_{i}\right) \mid 1<i<3\right\rangle$ acts transitively on $X$ and hence $\left\langle Z O_{p} H\left(x_{i}\right) \mid 1 \leqslant i \leqslant 3\right\rangle=\left\langle Z O_{p} H(x) \mid x \in X\right\rangle$. If $\left.q\right\rangle 2$, we have $\left\langle Z O_{p} H(x) \mid x \in X\right\rangle=H$ since $H$ is simple; when $q=2,\left\langle Z O_{p} H(x) \mid x \in X\right\rangle=H^{\prime}$.

LEMMA 4. Let $P_{1}$ be a nontrivial subgroup of $Z O_{p} H(x)$ for some $x \in X$ and let $\mathcal{C}$ be the set of subgroups conjugate to $P_{1}$ in $H$. If $\left|P_{1}\right|>2$ or $q=2$, there exist $P_{2}$ and $P_{3} \in \mathcal{C}$ such that $\left\langle P_{1}, P_{2}, P_{3}\right\rangle=H^{\prime}$ (where $H=H^{\prime}$ if $\left.q\right\rangle 2$ ). If $\left|P_{1}\right|=2$ and $q\rangle 2$, there exist $P_{2}, P_{3}$ and $P_{4} \in \mathcal{C}$ such that $\left\langle P_{1}, P_{2}, P_{3}, P_{4}\right\rangle=H$.

Proof. Let $Y$ be the set of absolute points on some nonabsolute line through $x$. $H_{Y}$ induces $P G L(2, q)$ on $Y$. If $q$ is even, there exists, according to [7, (8.8.27)], a subgroup $P_{2} \in \mathcal{C}$ such that $\left\langle P_{1}, P_{2}\right\rangle^{Y}$ contains a dihedral group of order $2(q+1)$ which is maximal in $H_{Y}^{Y} \cong \operatorname{PSL}(2, q)$. If $\left|P_{1}\right|>2$ or $q=2$, we have $\left\langle P_{1}, P_{2}\right\rangle^{Y} \cong$ $\operatorname{PSL}(2, q)$. If $\left|P_{1}\right|=2$ but $q>2$, there is a subgroup $P_{4} \in \mathcal{C}, P_{4}<H(x)$, distinct from $P_{1}$; we have $\left\langle P_{1}, P_{2}, P_{4}\right\rangle^{Y} \cong P S L(2, q)$. If $q$ is odd, it follows from [6, (2.8.4)] if $q \neq 9$ and from $P S L(2,9) \cong A_{6}$ when $q=9$ that there exists a $P_{2} \in \mathcal{C}$ such that $\left\langle P_{1}, P_{2}\right\rangle^{Y} \cong \operatorname{PSL}(2, q)$ since any two $p$-elements of $\operatorname{PSL}(2, q)$ are conjugate in $P G L(2, q)$ and $A_{6}$ can be generated by two of its 3-elements.

Now suppose that for some subgroup $A$ of $H_{Y}, A^{Y} \cong P S L(2, q), q$ even or odd. Then $Z O_{p} H(y) \leqslant A$ for every $y \in Y$. If $P_{3}$ is any subgroup in $C$ whose absolute fixed point does not lie on $Y$, then $g(Y) \neq Y$ for every nontrivial element $g$ in $P_{3}$ and so $\left\langle A, P_{3}\right\rangle$ contains $Z O_{p} H(z)$ for at least three noncollinear points $z \in X$.

In the proof of Theorem 3 we will need to deal with an arbitrary elementary abelian subgroup of $O_{p} H(x)$. If $p=2$, every such subgroup is contained in $Z O_{p} H(x)$. For odd $p$, this is not so.

LeMma 5. Let $p \neq 2$, let $P_{1}$ be a nontrivial elementary abelian subgroup of $O_{p} H(x)$ for some $x \in X$ and let $\mathcal{C}$ be the set of subgroups conjugate to $P_{1}$ in $H$. Then $\left|P_{1}\right|<q^{2}$. If $P_{1} \cap Z O_{p} H(x)=1$ then $\left|P_{1}\right|<q$ and there exist $P_{2}, P_{3}$ and $P_{4} \in \mathcal{C}$ such that $\left\langle P_{1}, P_{2}, P_{3}, P_{4}\right\rangle=H$. If $\left|P_{1}\right|>q$ then there exists a single subgroup $P_{2}$ such that $\left\langle P_{1}, P_{2}\right\rangle=H$.

Proof. It is easily checked that $\left|P_{1}\right| \leqslant q^{2}$ and $\left|P_{1}\right|<q$ if $P_{1} \cap Z O_{p} H(x)=1$. Suppose first that $\left|P_{1}\right|>q$ so that $P_{1} \cap Z O_{p} H(x) \neq 1$. Given any nonabsolute line $L$ through $x$, we may choose $P_{2} \in \mathcal{C}$ such that $\left\langle P_{1}, P_{2}\right\rangle$ induces $\operatorname{PSL}(2, q)$ on $Y$, where $Y=X \cap L$. Since $\left|P_{1}\right|>q, P_{1} \leqslant Z O_{p} H(x)$ and so $P_{1}$ contains elements which do not map $Y$ to itself. Hence $\left\langle P_{1}, P_{2}\right\rangle$ contains $Z O_{p} H(z)$ for at least three noncollinear points $z \in X$. Thus $\left\langle P_{1}, P_{2}\right\rangle=H$. Now suppose $P_{1} \cap Z O_{p} H(x)=1$. Choose any nontrivial element $a \in P_{1}$. There exists an homology $h \in H(x)$ such that $a$ and $a^{h}$ do not commute. Let $P_{2}=P_{1}^{h}$. Then $1 \neq\left[a, a^{h}\right] \in\left(O_{p} H(x)\right)^{\prime}=$ $Z O_{p} H(x)$; thus $\left\langle P_{1}, P_{2}\right\rangle \cap Z O_{p} H(x) \neq 1$. It follows now just as in the previous case that there exists a subgroup $P$ conjugate to $\left\langle P_{1}, P_{2}\right\rangle$ in $H$ such that $\left\langle P_{1}, P_{2}, P\right\rangle=H$.

$P \Gamma U\left(3, q^{2}\right)$ is the subgroup of $P \Gamma L\left(3, q^{2}\right)$ consisting of those elements which commute with the polarity $\delta$. Let $K=P \Gamma U\left(3, q^{2}\right)$. Then $K \approx \operatorname{aut}(H)$ and for each $x \in X, O_{p} H(x)=O_{p} K(x)$. If $H \leqslant L \leqslant K$, then $p$ is the only local prime of $L(x)$, i.e., the only prime such that $O_{p} L(x) \neq 1$. 
We now begin the actual proof of Theorem 3. Suppose $\Gamma$ and $G$ fulfill the hypotheses. According to [8, (4.9)] if $q=2$ and [8, (3.5), condition (1)] if $q>2$, $G_{1}(x)=G_{1}^{\prime}(x)$. Suppose that $G_{1}(x, y) \neq 1$. Recalling our previous remarks, we note that [8, (4.11)] continues to hold when $G^{V(\Gamma)}$ is imprimitive if $\Gamma$ is assumed to be undirected and connected. Thus $G_{1}(x, y)$ is a $p$-group since $p$ is the only local prime of $G(x, y)^{\Gamma(x)}$.

Let $u \in \Gamma(x)$ or $\Gamma^{\prime}(x)$ be arbitrary. We claim that $Z O_{p} G(x, u)$ is contained in $Z O_{p} G(x)$. Suppose the contrary. Since $O_{p}\left(G(x)^{\Gamma(x)}\right)=1, O_{p} G(x)=O_{p} G_{1}(x)=$ $O_{p} G(x, u) \cap G_{1}(x)$. It follows that $Z O_{p} G(x, u) \nless G_{1}(x)$. Since $G_{1}(x)=G_{1}^{\prime}(x)$, we can find $a \in Z O_{p} G(x, u), z \in \Gamma(x)$ and $w \in \Gamma^{\prime}(x)$ such that $a \notin G(z)$ and $a \notin$ $G(w)$. Since $G_{1}(x, z) \leqslant G_{1}(x), G_{1}(x, z) \leqslant O_{p} G_{1}(x) \leqslant O_{p} G(x, u)$ and hence $G_{1}(x, z)$ $=a G_{1}(x, z) a^{-1}=G_{1}(x, a(z))$. Therefore $G_{1}(x, z) \leqslant\langle G(x, z), G(x, a(z))\rangle$. Since $G(x)^{\Gamma(x)}$ is primitive but not regular and $a(z) \neq z,\langle G(x, z), G(x, a(z))\rangle=G(x)$. Thus $G_{1}(x, z) \varangle G(x)$. Similarly, $G_{1}(w, x) \varangle G(x)$. If $b \in G$ is an element mapping $(w, x)$ to $(x, z)$ then $G_{1}(x, z)=b G_{1}(w, x) b^{-1} \leqslant b G(x) b^{-1}=G(z)$. It follows that $G_{1}(x, z) \diamond\langle G(x), G(z)\rangle$ although $G_{1}(x, z) \neq 1$, a contradiction. Thus $Z O_{p} G(x, u)$ $<Z O_{p} G(x)$ as claimed.

Let $\Omega_{1}$ be the functor which assigns to a $p$-group the subgroup generated by its elements of order $p$. Let $V=\left\langle\Omega_{1} Z O_{p} G(x, u) \mid u \in \Gamma(x) \cup \Gamma^{\prime}(x)\right\rangle$. By the previous paragraph, $V \leqslant \Omega_{1} Z O_{p} G(x)$. Let $C(V)=C_{G(x)}(V)$, the centralizer of $V$ in $G(x)$, and suppose that $C(V) \nless G_{1}(x)$. Since $G_{1}(x)=G_{1}^{\prime}(x)$, we can find $a \in C(V)$, $z \in \Gamma(x)$ and $w \in \Gamma^{\prime}(x)$ such that $a \notin G(z)$ and $a \notin G(w)$. Since $\Omega_{1} Z O_{p} G(x, z)<$ $V, \quad \Omega_{1} Z O_{p} G(x, z)=a \Omega_{1} Z O_{p} G(x, z) a^{-1}=\Omega_{1} Z O_{p} G(x, a(z))$ and thus $\Omega_{1} Z O_{p} G(x, z) \preccurlyeq\langle G(x, z), G(x, a(z))\rangle=G(x)$. Similarly, $\Omega_{1} Z O_{p} G(w, x) \triangleleft G(x)$. Conjugating $\Omega_{1} Z O_{p} G(w, x)$ by an element mapping $(w, x)$ to $(x, z)$, we conclude that $\Omega_{1} Z O_{p} G(x, z) \triangleleft G(z)$. Thus $\Omega_{1} Z O_{p} G(x, z) \triangleleft\langle G(x), G(z)\rangle$ and so $\Omega_{1} Z O_{p} G(x, z)=1$ although $1 \neq G_{1}(x, z) \leqslant O_{p} G(x, z)$, a contradiction. It follows that $C(V)<G_{1}(x)$.

Let $m$ denote the functor which assigns to a $p$-group the maximal order of an elementary abelian subgroup and $J_{1}$ the functor which assigns to a $p$-group the subgroup generated by all its elementary abelian subgroups of this order. Suppose $J_{1} O_{p} G(x, y)<G_{1}(x)$. Then $J_{1} O_{p} G(x, y) \leqslant O_{p} G(x, y) \cap G_{1}(x)=O_{p} G(x)$ and hence $m O_{p} G(x, y)=m O_{p} G(x)$ and $J_{1} O_{p} G(x, y)=J_{1} O_{p} G(x)$. Let $w \in \Gamma^{\prime}(x)$ be arbitrary. Since $G$ acts transitively on $K(\Gamma), m O_{p} G(w, x)=m O_{p} G(x, y)=$ $m O_{p} G(x)$. Since $O_{p} G(x) \leqslant O_{p} G(w, x), J_{1} O_{p} G(x) \leqslant J_{1} O_{p} G(w, x)$. Since $\left|J_{1} O_{p} G(x)\right|$ $=\left|J_{1} O_{p} G(x, y)\right|=\left|J_{1} O_{p} G(w, x)\right|, \quad J_{1} O_{p} G(x)=J_{1} O_{p} G(w, x)$. Conjugating $J_{1} O_{p} G(w, x)$ by an element mapping $(w, x)$ to $(x, y)$, we see that $J_{1} O_{p} G(x, y) \leqslant$ $G(y)$. Thus $J_{1} O_{p} G(x, y) \leqslant\langle G(x), G(y)\rangle$ and so $J_{1} O_{p} G(x, y)=1$ although $O_{p} G(x, y)$ $\neq 1$, a contradiction. It follows that $J_{1} O_{p} G(x, y) \nless G_{1}(x)$.

Choose $P_{1}$ among those elementary abelian subgroups of $O_{p} G(x, y)$ of order $m O_{p} G(x, y)$ not contained in $G_{1}(x)$ and let $P_{0}=P_{1} \cap G_{1}(x)$. Since $V<$ $\Omega_{1} Z O_{p} G(x)$ and $P_{0}<O_{p} G(x, y) \cap G_{1}(x)=O_{p} G(x), P_{0} V$ is elementary abelian. Hence $\left|P_{0} V\right|<\left|P_{1}\right|$. Since $P_{1}$ is abelian, $P_{0} \cap V<C_{V}\left(P_{1}\right)$. Therefore $\left|P_{1} / P_{0}\right| \geqslant$ $\left|P_{0} V / P_{0}\right|=\left|V / P_{0} \cap V\right| \geqslant\left|V / C_{V}\left(P_{1}\right)\right| . P_{1} / P_{0}$ is isomorphic to a nontrivial elementary abelian $p$-subgroup of $O_{p}\left(G(x, y)^{\Gamma(x)}\right)$. Let $\left|P_{1} / P_{0}\right|=p^{m}$. Let $t$ be an 
integer such that there exist subgroups $P_{2}, \ldots, P_{t}$ conjugate to $P_{1}$ in $G(x)$ such that, with $A=\left\langle P_{1}, \ldots, P_{t}\right\rangle, A^{\Gamma(x)} \cong P S U\left(3, q^{2}\right)^{\prime}$. Since $P_{i}$ is conjugate to $P_{1}$, $\left|V / C_{V}\left(P_{i}\right)\right|=\left|V / C_{V}\left(P_{1}\right)\right|$ for $2 \leqslant i \leqslant t$; hence $\left|V / C_{V}(A)\right| \leqslant\left|V / C_{V}\left(P_{1}\right)\right|^{t} \leqslant$ $\left|P_{1} / P_{0}\right|^{t}=p^{m t}$. Let $W=V / C_{V}(A)$ and $D=C_{A}(W)$. $D$ is normal in $A$. If $D \$$ $\mid G_{1}(x), D$ contains elements of order prime to $p$ not in $G_{1}(x)$. By [6, (5.3.2)], these elements lie in $C(V)$. This contradicts $C(V) \leqslant G_{1}(x)$. Hence $D<G_{1}(x)$. Since $A / D$ is faithfully represented on $W$, we have $A / D \leqslant G L(W)$ and thus $q^{3}+$ 1 ||$A^{\Gamma(x)}|||A / D||| G L(W) \mid$ and so $q^{3}+1$ divides $\left(p^{m t}-1\right)\left(p^{m t-1}-1\right) \cdots(p$ - 1).

Since $p^{m}$ is the order of a group isomorphic to an elementary abelian $p$-subgroup of $\operatorname{PSU}\left(3, q^{2}\right), p^{m} \leqslant q$ if $q$ is even and $p^{m} \leqslant q^{2}$ if $q$ is odd. If $q=2$ (and thus $p^{m}=2$ ), then according to Lemma 4 we can take $t=3$. This implies that $2^{3}+1$ divides $\left(2^{3}-1\right)\left(2^{2}-1\right)$ which is not true. Thus $q>2$. By [11, p. 283], there exists a prime $\pi$ dividing $q^{6}-1$ but not $p^{v}-1$ for any $v<6 n$ where $q=p^{n}$. In particular, $\pi$ divides $q^{3}+1=\left(q^{6}-1\right) /\left(q^{3}-1\right)$. Hence $\pi$ divides $\left(p^{m t}-1\right)\left(p^{m t-1}-\right.$ 1) $\cdots(p-1)$ and so $6 n \leqslant m t$. If $p^{m}>q$ then according to Lemma 5 we can take $t=2$ and so $6 n \leqslant 2 m$. Thus $p^{3 n} \leqslant p^{m}$. This contradicts $p^{m} \leqslant q^{2}$. It follows that $p^{m}<q$, i.e., $m \leqslant n$. According to Lemmas 4 and 5 , we can take $t \leqslant 4$. Thus $6 n<m t<4 m \leqslant 4 n$. With this contradiction, the proof of Theorem 3 is complete.

In conclusion, we note that to show that our proof of Theorem 3 remains valid if $\operatorname{PSU}\left(3, q^{2}\right)$ is replaced by $S z(q)$ or ${ }^{2} G_{2}(q)$, it is necessary only to prove an appropriate version of Lemmas 4 and 5 . I leave the case ${ }^{2} G_{2}(q)$ as a problem. For the Suzuki groups, the following result, a corollary of [10, Theorem 9], is easily seen to suffice.

Lemma 6. Let $P_{1}$ be a nontrivial elementary abelian 2-subgroup of $S z(q)$. Then there exists a subgroup $P_{2}$ conjugate to $P_{1}$ such that $\left\langle P_{1}, P_{2}\right\rangle$ contains a dihedral group of order $2(q-1)$ if $q>2$, of order 10 if $q=2$, which is maximal in $S z(q)$ (so that $\left\langle P_{1}, P_{2}\right\rangle=S z(q)$ if $\left.\left|P_{1}\right|>2\right)$.

\section{REFERENCES}

1. M. Bürker and W. Knapp, Zur Vermutung von Sims über primitive Permutationsgruppen. II, Arch. Math. 27 (1976), 352-359.

2. U. Dempwolff, A factorization lemma and an application, Arch. Math. 27 (1976), 18-21.

3. __ A factorization lemma and an application. II, Arch. Math. 27 (1976), 476-479.

4. G. Glauberman, Weakly closed elements of Sylow subgroups, Math. Z. 107 (1968), 1-20.

5. __ A characteristic subgroup of a p-stable group, Canad. J. Math. 20 (1968), 1101-1135.

6. D. Gorenstein, Finite groups, Harper and Row, New York and London, 1968.

7. B. Huppert, Endliche Gruppen. I, Springer-Verlag, Berlin and New York, 1967.

8. W. Knapp, On the point stabilizer in a primitive permutation group, Math. Z. 133 (1973), 137-168.

9. C. Sims, Graphs and finite permutation groups, Math. Z. 95 (1967), 76-86.

10. M. Suzuki, On a class of doubly transitive groups, Ann. of Math. 75 (1962), 105-145.

11. K. Zsigmondy, Zur Theorie der Potenzreste, Montash. Math. Phys. 3 (1892), 265-284.

Mathematisches Institut, Freie Universität Berlin, Königin-Luise-Strasse 24-26, D-1000 BerLin 33, West Germany 